\title{
Hierarchical
}

\section{Classification of Real Life Documents}

\author{
Ke Wang ${ }^{*}$, Senqiang Zhou ${ }^{\dagger}, Y u H e^{\ddagger}$
}

\section{Introduction}

Two features have successfully made on-line information comprehensible and accessible to people: hierarchically structured classes where topics are organized into a hierarchy of increasing specificity, and multi-classed documents where a document is classified into all relevant classes. One such information source is Yahoo! where a document on Dance, for example, could be reached from both Arts : Performing_Arts and Recreation topics in the topic hierarchy. The hierarchical feature of classes allows information to be examined and browsed at various topic specificities, and the multi-class feature allows information to be accessed from all related topics. However, most document classification techniques assume that there is a flat class space and each document has one class. The documents classified by such techniques are difficult to browse and access by people, especially when there are many classes such as in Yahoo!. In this paper, we propose a new technique for automatic classification of documents to address these real life requirements. This raises several research issues. We use Yahoo! for explanation.

1. Misclassification is non-symmetric. Misclassifying an ads on topic Travel into topic Outdoors is less erroneous than misclassifying it into topic Software. Indeed, the fact that many ads belong to both Travel and Outdoors, but few belong to both Travel and Software suggests that Travel is more similar to Outdoor than to Software. This feature becomes more prevailing in a hierarchical class space where some classes are more general than others.

\footnotetext{
*Simon Fraser University

${ }^{\dagger}$ Simon Fraser University

$\ddagger$ National University of Singapore
} 
2. Documents are multi-classed. A document is typically classified into all relevant classes. Traditional classification fails to do so because each training document is allowed to be associated with only one class. One solution to multi-classification is to build a classifier for each possible class and classify a new document by going through every classifier. In a sparsed class space where a document belongs to a small number of classes from a large class space, this approach will construct too many classifiers. Further, such an independent classification for each class does not take into account the similarity and hierarchical structure of classes as discussed above.

3. The sparse class space. For $k$ classes, a document could be associated potentially with any one of the $2^{k}-1$ subsets of classes. Even not so large $k$ will create a very sparse class space in multi-class classification. This sparseness makes the learning task of automatic classification difficult because there may not be enough training documents. However, unlike traditional classification, classes share similarities as discussed above. Exploring such similarities opens up new channels to deal with the sparsity problem of the class space.

In summary, classes of documents interact by being a generalization of one another and classifying common documents. Traditional classification techniques fail to recognize such interactions. In this paper, we regard classes as objects whose similarity can be measured, and the goal of classification is to determine the set of "relevant" classes under this measure. We consider training documents of the form $\left\{t_{1}, \ldots, t_{n} \mid C_{1}, \ldots, C_{k}\right\}$, where $t_{1}, \ldots, t_{n}$ are terms (keywords or phrases) from a given universe and $C_{1}, \ldots, C_{k}$ are classes from a given class hierarchy. $\left\{C_{1}, \ldots, C_{k}\right\}$ is called a classset. Given a collection of training documents, our task is to construct a classifier, consisting of rules of the form $\left\{t_{i_{1}}, \ldots, t_{i_{p}}\right\} \rightarrow\left\{C_{i_{1}}, \ldots, C_{i_{q}}\right\}$, that assigns a "good" classset $\left\{C_{i_{1}}, \ldots, C_{i_{q}}\right\}$ to a given new document. There are two contributions:

1. We define a new notion of similarity between two classsets using the similarity of the documents belonging to these classsets. The intuition is that two classsets are similar exactly when their classified documents are similar. Indeed, if two classsets classify many documents in common, the chance that they are similar topics is high, and misclassification from one to the other is less erroneous. We believe that this notion captures the essence of class similarity.

2. We construct a classifier using the proposed class similarity. A major challenge is the search of classification rules of the form $\left\{t_{i_{1}}, \ldots, t_{i_{p}}\right\} \rightarrow\left\{C_{i_{1}}, \ldots, C_{i_{q}}\right\}$, where $t_{i_{j}}$ are terms and $C_{i_{j}}$ are classes, because there are many terms and classes. Our approach is to apply the association rule mining $[1,2]$ to generate such rules. We present an algorithm for selecting association rules to construct a classifier. Yahoo!.

We evaluate this method using the documents in ACM Digital Library and 


\section{Related work}

With few exceptions, most classification systems assume that all classes are at a flat level and each document is labeled by one class [5, 6, 9]. Recently, hierarchically structured classes were examined in $[3,4,7]$ where classes are organized into a hierarchy of increasing specificity and a document is labeled by one class in the hierarchy. Though a document belonging to a child class is automatically considered as belonging to a parent class, a document is not allowed to belong to two classes not on a generalization path in the hierarchy, like Arts : Performing_Arts and Recreation in Yahoo!.

Related to multi-classification of documents is the problem of transforming source terms to target terms for a collection of documents [8]. Each training document is a pair $\langle s, t\rangle$ where $s$ is a set of source terms and $t$ is a set of target terms. The goal of the transformation is finding the transformation matrix from source terms to target terms that minimizes the total error for a collection of documents. In our terminology, target terms correspond to classes of a document and the transformation matrix corresponds to a classifier. [8] solves this problem as the Linear Least Squares Fit that performs the transformation using the standard singular value decomposition. That approach does not address the similarity and hierarchical nature of target terms. Also, the singular value decomposition is computationally expensive, in order $N^{2} \times k^{3}$, where $N$ is the number of terms and documents and $k$ is the number of terms. For ACM Digital Library, $N$ and $k$ could be tens of thousands or even more.

The extended kNN [9] returns the set of classes of the $k$ training documents nearest to the given document as the relevant classes. We will compare our method with kNN. Some classifiers such as decision tree [5] return a class distribution for a given document, in the form of the probability of each class. However, these classifiers do not consider the similarity of classes and the hierarchical structure of the class space.

\section{A new class similarity}

We measure the similarity of two classsets by the similarity of the training documents belonging to them. Let us describe this idea formally. Consider a document $d$ belonging to $k$ classes $C_{1}, \ldots, C_{k}$, or simply belonging to the classset $C S=\left\{C_{1}, \ldots, C_{k}\right\}$. Clearly, $d$ also belongs to the classes that are more "general" than $C_{i}, 1 \leq i \leq k$. These general classes are the ancestors of $C_{i}$ in the class hierarchy. Let $A n c(C S)$ denote the set of classes in $C S$ plus their ancestors.

Definition 1. Consider two classsets $C S_{1}$ and $C S_{2}$. We say that $C S_{1}$ is more general than $C S_{2}$ if $A n c\left(C S_{1}\right) \subseteq A n c\left(C S_{2}\right)$. We say that a document d is covered by a classset $C S$ if $C S$ is more general than the classset of $d$. The coverage of $C S$, denoted by Cover(CS), is the set of all documents covered by CS.

For example, if Dance is a parent of Fast_Dance, $\{$ Dance $\}$ is more general than $\{$ Fast_Dance, Music $\}$ because Anc( $\{$ Dance $\}) \subseteq$ Anc $(\{$ Fast_Dance, Music $\})$. 
The following equivalence holds (we omit the straightforward proof):

Lemma 2. Consider a classset CS and a document $d$ with classset $C S_{d}$. The

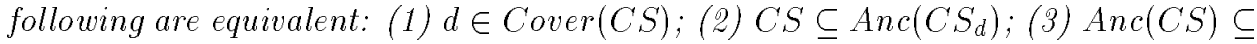
$\operatorname{Anc}\left(C S_{d}\right)$.

Lemma 3. Cover $\left(C S_{1}\right) \cap \operatorname{Cover}\left(C S_{2}\right)=\operatorname{Cover}\left(C S_{1} \cup C S_{2}\right)$.

Proof: Let $d \in$ Cover $\left(C S_{1}\right) \cap$ Cover $\left(C S_{2}\right)$. From Lemma 2, CS $S_{1} \subseteq \operatorname{Anc}\left(C S_{d}\right)$ and $C S_{2} \subseteq A n c\left(C S_{d}\right)$, where $C S_{d}$ denotes the classset of $d$, therefore, $\bar{C} S_{1} \cup C S_{2} \subseteq$ Anc $\left(C S_{d}\right)$. From Lemma 2, this implies that $d \in \operatorname{Cover}\left(C S_{1} \cup C S_{2}\right)$. This shows that $\operatorname{Cover}\left(C S_{1}\right) \cap \operatorname{Cover}\left(C S_{2}\right) \subseteq \operatorname{Cover}\left(C S_{1} \cup C S_{2}\right)$. To show the other containment, let $d \in \operatorname{Cover}\left(C S_{1} \cup C S_{2}\right)$. From Lemma 2, $C S_{1} \cup C S_{2} \subseteq \operatorname{Anc}\left(C S_{d}\right)$, and thus, $C S_{i} \subseteq$ Anc $\left(C S_{d}\right), i=1,2$. Then, from Lemma 2, $d$ is in Cover $\left(C S_{i}\right)$.

The dissimilarity of two classsets $C S_{1}$ and $C S_{2}$ is defined as the normalized difference of their coverages:

$$
E\left(C S_{1}, C S_{2}\right)=\frac{\left|\operatorname{Cover}\left(C S_{2}\right)-\operatorname{Cover}\left(C S_{1}\right)\right|+\left|\operatorname{Cover}\left(C S_{1}\right)-\operatorname{Cover}\left(C S_{2}\right)\right|}{\left|\operatorname{Cover}\left(C S_{1}\right) \cup \operatorname{Cover}\left(C S_{2}\right)\right|}
$$

$|x|$ denotes the number of elements in a set $x . E\left(C S_{1}, C S_{2}\right)$ is in the range [0,1]. The similarity of $C S_{1}$ and $C S_{2}$ is defined as $1-E\left(C S_{1}, C S_{2}\right)$. From Lemma 3 , we rewrite definition (1) as

$$
E\left(C S_{1}, C S_{2}\right)=\frac{\left|\operatorname{Cover}\left(C S_{1}\right)\right|+\left|\operatorname{Cover}\left(C S_{2}\right)\right|-2\left|\operatorname{Cover}\left(C S_{1} \cup C S_{2}\right)\right|}{\left|\operatorname{Cover}\left(C S_{1}\right)\right|+\left|\operatorname{Cover}\left(C S_{2}\right)\right|-\left|\operatorname{Cover}\left(C S_{1} \cup C S_{2}\right)\right|}
$$

Therefore, to compute $E\left(C S_{1}, C S_{2}\right)$, we need only to compute the coverage of $C S_{1}, C S_{2}, C S_{1} \cup C S_{2}$.

We say that a document $d$ matches a rule $T \rightarrow C S$, or vice versa, if $d$ contains all the terms in $T$. Let $M a t c h(T \rightarrow C S)$ denote the set of training documents that match $T \rightarrow C S$. The generalized confidence of $T \rightarrow C S$ is defined as

$$
\operatorname{Conf}_{g}(T \rightarrow C S)=\frac{\operatorname{Match}(T \rightarrow C S)-\Sigma_{d} E\left(C S_{d}, C S\right)}{\operatorname{Match}(T \rightarrow C S)}
$$

where $d$ ranges over the elements of $M a t c h(T \rightarrow C S)$, and $C S_{d}$ is the classset of $d$. Intuitively, Con $f_{g}(T \rightarrow C S)$ measures the average similarity between $C S$ and the classsets of the documents that match $T \rightarrow C S$. If $E\left(C S_{1}, C S_{2}\right)$ is binary, i.e., 1 or $0, \Sigma_{d} E\left(C S_{d}, C S\right)$ degenerates to the number of training documents that match $T \rightarrow C S$ but do not belong to $C S$, and $C o n f_{g}(T \rightarrow C S)$ degenerates to the standard confidence [1]. In this sense, $\operatorname{Con} f_{g}(T \rightarrow C S)$ is a generalization of the standard confidence in the presence of class similarity.

Example 1. Consider the database of 6 documents $d_{1}, \ldots, d_{6}$ and the class hierarchy in Figure 1. We write $B C$ to mean classset $\{B, C\}$, and similarly for the others. Cover $(B)=\left\{d_{1}, d_{2}, d_{3}, d_{4}, d_{6}\right\}$ because $B$ is contained in Anc $\left(C S_{d_{i}}\right)$ for $i=1,2,3,4,6$ (from Lemma 2). Figure 1(c) lists the coverage of some classsets. For example, $E(B, C)$ is computed as follows: 


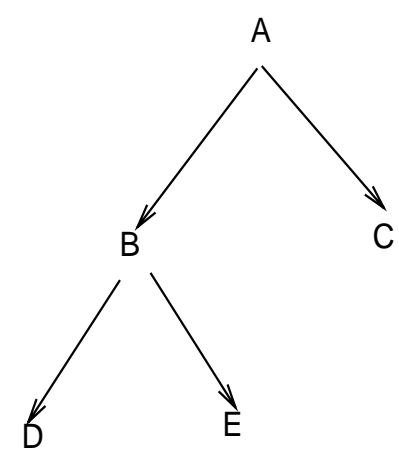

(a) Class hierarchy

\begin{tabular}{|l|l|l|}
\hline id & items & classset \\
\hline$d_{1}$ & $k_{1}$ & $\mathrm{~B}$ \\
\hline$d_{2}$ & $k_{1}, k_{2}$ & $\mathrm{BC}$ \\
\hline$d_{3}$ & $k_{1}, k_{2}$ & $\mathrm{BC}$ \\
\hline$d_{4}$ & $k_{2}$ & $\mathrm{CD}$ \\
\hline$d_{5}$ & $k_{3}$ & $\mathrm{C}$ \\
\hline$d_{6}$ & $k_{3}$ & $\mathrm{CE}$ \\
\hline
\end{tabular}

(b) Documents

\begin{tabular}{|l|l|}
\hline $\mathrm{CS}$ & Cover $(C S)$ \\
\hline $\mathrm{B}$ & $d_{1}, d_{2}, d_{3}, d_{4}, d_{6}$ \\
\hline $\mathrm{BC}$ & $d_{2}, d_{3}, d_{4}, d_{6}$ \\
\hline $\mathrm{C}$ & $d_{2}, d_{3}, d_{4}, d_{5}, d_{6}$ \\
\hline $\mathrm{CD}$ & $d_{4}$ \\
\hline $\mathrm{CE}$ & $d_{6}$ \\
\hline
\end{tabular}

(c) Coverage

Figure 1. An example

Cover $(B)-\operatorname{Cover}(C)=\left\{d_{1}, d_{2}, d_{3}, d_{4}, d_{6}\right\}-\left\{d_{2}, d_{3}, d_{4}, d_{5}, d_{6}\right\}=\left\{d_{1}\right\}$ Cover $(C)-\operatorname{Cover}(B)=\left\{d_{2}, d_{3}, d_{4}, d_{5}, d_{6}\right\}-\left\{d_{1}, d_{2}, d_{3}, d_{4}, d_{6}\right\}=\left\{d_{5}\right\}$ Cover $(B) \cup$ Cover $(C)=\left\{d_{1}, d_{2}, d_{3}, d_{4}, d_{5}, d_{6}\right\}$

$$
E(B, C)=\frac{\left|\left\{d_{1}\right\}\right|+\left|\left\{d_{5}\right\}\right|}{\left|\left\{d_{1}, d_{2}, d_{3}, d_{4}, d_{5}, d_{6}\right\}\right|}=\frac{2}{6}=0.33
$$

Table 1 shows the dissimilarity between some classsets.

Con $f_{g}\left(k_{1} \rightarrow B\right)$ is computed as follows. $k_{1}$ is found in $d_{1}, d_{2}, d_{3}, 1$ with no error and 2 with error $E(B, B C)$. So we have

$$
\operatorname{Conf}_{g}\left(k_{1} \rightarrow B\right)=\frac{3-2 \times E(B, B C)}{3}=\frac{3-2 \times 0.2}{3}=0.87
$$

Similarly,

$$
\begin{gathered}
C o n f_{g}\left(k_{2} \rightarrow B C\right)=\frac{3-E(B C, C D)}{3}=\frac{3-0.75}{3}=0.75 \\
\operatorname{Conf}_{g}\left(k_{3} \rightarrow C\right)=\frac{2-E(C E, C)}{2}=\frac{2-0.8}{2}=0.6 \\
\operatorname{Conf}_{g}\left(k_{2} \rightarrow C\right)=\frac{3-2 \times E(B C, C)-E(C D, C)}{3}=\frac{3-2 \times 0.2-0.8}{3}=0.6
\end{gathered}
$$




\begin{tabular}{|l||l|l|l|l|l|}
\hline & B & BC & C & CD & CE \\
\hline \hline B & 0 & 0.2 & 0.33 & 0.8 & 0.8 \\
\hline BC & 0.2 & 0 & 0.2 & 0.75 & 0.75 \\
\hline C & 0.33 & 0.2 & 0 & 0.8 & 0.8 \\
\hline CD & 0.8 & 0.75 & 0.8 & 0 & 1 \\
\hline CE & 0.8 & 0.75 & 0.8 & 1 & 0 \\
\hline
\end{tabular}

Table 1. $E\left(C S_{1}, C S_{2}\right)$

\section{Construction of classifiers}

There are four steps in constructing a classifier. First, we generate association rules of the form $T \rightarrow C S$, where $T$ is a set of terms and $C S$ is a classset, that satisfy the user-specified minimum support and minimum confidence, as in [1, 2]. Second, we rank rules to determine the classification rule of a document. Third, we remove the rules that incorrectly classify many training documents. Fourth, we cut off the ranked list to minimize the overall classification error. Let us explain each step in details.

\subsection{Step 1: Find association rules}

We generate all association rules of the form $T \rightarrow C S$ that satisfy some userspecified minimum support and minimum confidence. The algorithm is basically that of [2]. There are several differences.

First, each frequent k-itemset ([2]'s terminology), $k>1$, contains at least one term and at least one class. Each such frequent itemset TCS represents a rule $T \rightarrow C S$, where $T$ is a set of terms and $C S$ is a classset. For $k>2$, every frequent $k$-itemset of this form can be constructed using two frequent $(k-1)$-itemsets of the same form, like in the Apriori [2], adding either one term in $T$ or one class in $C S$. This restricted form reduces substantially the number of itemsets generated. Second, we use the generalized confidence of rule $T \rightarrow C S$ as in defined by Equation 3 , not the standard confidence in [2]. $E\left(C S, C S_{d}\right)$ in Equation 3 is not available from frequent itemsets. We need two database scans to compute generalized confidence of all rules.

1. In the first scan, we compute $\mid$ Cover $(C S) \mid$ for $C S$, where $C S$ is either a classset $C S_{d}$ appearing in some training documents, or a classset $C S_{r}$ appearing in some rules found, or $C S_{d} \cup C S_{r}$. In particular, for each document $d$ scanned, we increment $\mid$ Cover $(C S) \mid$ for $C S$ if $C S \subseteq A n c\left(C S_{d}\right)$, where $C S_{d}$ is the classset of $d$. Then, we compute $E\left(C S_{d}, \bar{C} S_{r}\right)$ based on Equation (1) for classset $C S_{d}$ of training documents $d$ and classset $C S_{r}$ in rules $r$ found. 
2. In the second scan, we compute $\operatorname{Con} f_{g}(T \rightarrow C S)$ for every rule $T \rightarrow C S$ generated. In particular, for each training document $d$, we find all matching rules $T \rightarrow C S$, i.e., $T \subseteq d$, increment $M$ atch $(T \rightarrow C S)$ and add $E\left(C S_{d}, C S\right)$ to $\Sigma_{d} E\left(C S_{d}, C S\right)$. At the end of the scan, $C o n f_{g}(T \rightarrow C S)$ is computed by Equation (3).

\subsection{Step 2: rank the rules}

A document could match more than one rule, one of which is chosen to classify the document. We propose the most-confident-first (MCF) principle to determine the classification rule of each document: a document is classified by the matching rule that has the highest generalized confidence (breaking a tie arbitrarily). If we rank all rules by generalized confidence, the MCF principle says that the first matching rule in the ranked list is chosen as the classification rule for a document. The generalized confidence of a rule $T \rightarrow C S$ measures the average similarity between $C S$ and the classsets of the documents matched by the rule. By choosing the matching rule with highest generalized confidence, a document is assigned the most similar classset among all the matching rules. Under the MCF principle, among all the rules $T \rightarrow C S$ with the same LHS $T$, only the rule with the highest generalized confidence will actually classify some documents, therefore, needs to be kept.

\subsection{Step 3: remove rules of low accuracy}

Let $D$ be the set of training documents classified by rule $T \rightarrow C S$ under the MCF principle. If $D$ is non-empty, the accuracy of $T \rightarrow C S$ is defined as

$$
\operatorname{Accu}(T \rightarrow C S)=\frac{|D|-\operatorname{Error}(T \rightarrow C S)}{|D|}
$$

where $\operatorname{Error}(T \rightarrow C S)$ is the error of $T \rightarrow C S$ defined as

$$
\operatorname{Error}(T \rightarrow C S)=\Sigma_{d \in D} E\left(C S_{d}, C S\right)
$$

The accuracy of all rules can be computed by one scan of the documents, given that all errors $E\left(C S_{d}, C S\right)$ were computed in Step 1. We remove all rules with accuracy below a certain threshold because they contribute negatively to the overall accuracy. Note that $\operatorname{Con} f_{g}(T \rightarrow C S)$ is defined with respect to all documents that match the rule, whereas $A c c u(T \rightarrow C S)$ is defined with respect to the documents classified by the rule under the MCF principle.

Example 2. Consider the database in Example 1 and the error in Table 1. Let the minimum support be 2/6. In Step 1, the following rules above the minimum support are generated (we do not specify minimum confidence), ranked by generalized confidence:

$$
\begin{aligned}
& r_{1}: k_{1} \rightarrow B\left(\text { match } d_{1}, d_{2}, d_{3}, \operatorname{con} f_{g}=0.87\right) \\
& r_{2}: k_{2} \rightarrow B C\left(\text { match } d_{2}, d_{3}, d_{4}, \text { conf } f_{g}=0.75\right) \\
& r_{3}: k_{3} \rightarrow C\left(\text { match } d_{5}, d_{6}, \text { con } f_{g}=0.60\right) \\
& r_{4}: k_{2} \rightarrow C\left(\text { match } d_{2}, d_{3}, d_{4}, \operatorname{con} f_{g}=0.60\right)
\end{aligned}
$$


let $r_{1}, \ldots, r_{m}$ be the remaining rules after Step 3 , sorted by $C o n f_{g}\left(r_{i}\right)$

recompute $\operatorname{Error}\left(r_{i}\right)$ for $1 \leq i \leq m$

${ }^{*}$ compute the cutoff point $* /$

PrefixError $\left(r_{i}\right)=0$

foreach $i=1$ to $m$ do

PrefixError $\left(r_{i}\right)=$ PrefixEror $\left(r_{i-1}\right)+\operatorname{Error}\left(r_{i}\right)$

let Unclassifed $\left(r_{i}\right)$ be the set of training documents not classified by $r_{1}, \ldots, r_{i}$

let DefaultClass $\left(r_{i}\right)$ be the classset $C S$ that minimizes

$\Sigma_{\left.d \in \text { Unclassified( } r_{i}\right)} E\left(C S, C S_{d}\right)$

DefaultError $\left(r_{i}\right)=\Sigma_{d \in \text { Unclassified }\left(r_{i}\right)} E\left(\right.$ DefaultClass $\left.\left(r_{i}\right), C S_{d}\right)$

find the smallest $k$ that minimizes PrefixError $\left(r_{k}\right)+$ DefaultError $\left(r_{k}\right)$

return prefix $r_{1}, \ldots, r_{k}$ and default class Default Class $\left(r_{k}\right)$

Table 2. Step 4

Con $f_{g}$ is computed as in Example 1. In Step 2, we apply the MCF principle to determine the classification rule for each training document:

$r_{1}: k_{1} \rightarrow B$ (classify $\left.d_{1}, d_{2}, d_{3}, A c c u=0.87\right)$

$r_{2}: k_{2} \rightarrow B C$ (classify $d_{4}$, Accu $\left.=0.25\right)$

$r_{3}: k_{3} \rightarrow C$ (classify $d_{5}, d_{6}$, Accu $\left.=0.60\right)$

$r_{4}: k_{2} \rightarrow C$ (classify no document)

In Step 3, we compute the accuracy of rules. Accu( $\left(r_{1}\right)=$ Con $f_{g}\left(r_{1}\right)=0.87 . r_{2}$ classifies only $d_{4}$, so Accu $\left(r_{2}\right)=\frac{1-E(B C, C D)}{1}=0.25$. $r_{3}$ classifies all documents it matches, so Accu $\left(r_{3}\right)=C$ Con $f_{g}\left(r_{3}\right)=0.60 . r_{4}$ has no turn to classify any document. Suppose that we set the threshold of accuracy at 0.5, $r_{2}$ is removed, and $r_{4}$ now classifies $d_{4}$.

\subsection{Step 4: cut off the ranked list}

Finally, we cut off the ranked list of remaining rules to minimize the cutoff error. Let $r_{1}, \ldots, r_{m}$ be the ranked list of remaining rules. Suppose that we cut off the list after the first $i$ rules, $r_{1}, \ldots, r_{i}$. The cutoff error is PrefixError $\left(r_{i}\right)+$ De faultError $\left(r_{i}\right)$. PrefixError $\left(r_{i}\right)$ is the sum of the rule error Error $\left(r_{j}\right)$ for all rules $r_{j}, 1 \leq j \leq i$. DefaultError $\left(r_{i}\right)$ is the error caused by assigning the default classset to all the training documents not classified by any rule $r_{j}, 1 \leq j \leq i$. The default classset is chosen to minimize DefaultError $\left(r_{i}\right)$. Table 2 shows Step 4 . Since the rule error Error $\left(r_{j}\right)$ may have been changed by removing rules in Step 3, In Table 2, we first compute the rule error as in Step 3.

Example 3. We continue with Example 2. After removing $r_{2}$, the error of the remaining rules is computed as follows 


$$
\begin{aligned}
& \operatorname{Error}\left(r_{1}\right)=E\left(B, C S_{d_{1}}\right)+E\left(B, C S_{d_{2}}\right)+E\left(B, C S_{d_{3}}\right) \\
& \quad=E(B, B)+E(B, B C)+E(B, B C)=0.4 \\
& \operatorname{Error}\left(r_{3}\right)=E\left(C, C S_{d_{5}}\right)+E\left(C, C S_{d_{6}}\right)=E(C, C)+E(C, C E)=0.8 \\
& \operatorname{Error}\left(r_{4}\right)=E\left(C, C S_{d_{4}}\right)=E(C, C D)=0.8
\end{aligned}
$$

In Step 4, we determine the cutoff point of the remaining rules $\left\langle r_{1}, r_{3}, r_{4}\right\rangle$. For the shortest prefix \langle\rangle , the default classset is $B C$ (of $d_{2}$ ), which has the minimum error of 1.9. This is shown in the first row of Table 3. For prefix $\left\langle r_{1}\right\rangle$, the cutoff error is the sum of Error $\left(r_{1}\right)$ and the default error for unclassified documents $d_{4}, d_{5}, d_{6}$. The default classset is $C$ (of $d_{5}$ ), which gives the minimum default error, $E(C, C D)+E(C, C E)=1.6$. Thus, the cutoff error for $\left\langle r_{1}\right\rangle$ is $0.4+1.6=2.0$, as shown in the second row of Table 3. For prefix $\left\langle r_{1}, r_{3}\right\rangle$, the cutoff error is Error $\left(r_{1}\right)+E$ Error $\left(r_{3}\right)$ plus the default error for unclassified $d_{4}$. In this case the default classset is the classset of $d_{4}, C D$, with the default error of 0 . So the cutoff error for $\left\langle r_{1}, r_{3}\right\rangle$ is 1.2, shown in the third row of Table 3. At this point, since the default error is 0 , the cutoff error cannot be reduced by considering longer prefixes. Therefore, $\left\langle r_{1}, r_{3}\right\rangle$ is the shortest prefix that has the minimum cutoff error.

If we do not remove $r_{2}$ in Step 3. The error of each rule in $\left\langle r_{1}, r_{2}, r_{3}, r_{4}\right\rangle$ is

$$
\begin{aligned}
& \operatorname{Error}\left(r_{1}\right)=E\left(B, C S_{d_{1}}\right)+E\left(B, C S_{d_{2}}\right)+E\left(B, C S_{d_{3}}\right)=0.4 \\
& \operatorname{Error}\left(r_{2}\right)=E\left(B C, C S_{d_{4}}\right)=E(B C, C D)=0.75 \\
& \operatorname{Error}\left(r_{3}\right)=E\left(C, C S_{d_{5}}\right)+E\left(C, C S_{d_{6}}\right)=E(C, C)+E(C, C E)=0.8 \\
& \operatorname{Error}\left(r_{4}\right)=0
\end{aligned}
$$

Table 4 shows the computation of cutoff errors. In this case, the empty prefix $<>$ with the default classset $B$ gives the minimum cutoff error, 1.9. This is larger than that of the classifier $\left\langle r_{1}, r_{3}\right\rangle$ found earlier.

\section{Experiments}

We evaluate the effectiveness of the proposed method using the IBM Patent data and ACM Digital Library. For comparison, most traditional classification methods deal with data in the form of a table or assumes that a document belongs to one class. Such methods cannot work on the multi-classed documents here. We compare our method, denoted Coverage, with two methods, Confidence and kNN. Confidence is the same as Coverage except that it treats each classset as a new class in a flat class space, thus, ignoring the similarity of classset. This method ranks rules by the traditional confidence. Comparison with Confidence will reveal the effectiveness of the proposed similarity of classsets. kNN is the kNN extended with feature selection, which is highly competitive even compared with sophisiticated methods [9]. Given a new document, kNN uses the classes of the $k$ nearest training documents to predict the classset of the new document. The distance of these documents is used as a weight for their classes. One parameter of kNN is the feature threshold used by the feature selection. Another parameter of kNN is the cutoff threshold of class list. The kNN returns a list of ranked classes (by weight). We select the top classes that are 


\begin{tabular}{|l|l|l|l|l|l|}
\hline$\langle r 1, \ldots, r i\rangle$ & Error $(r i)$ & unclassified doc. & default classset & default error & cutoff error \\
\hline\langle\rangle & 0 & $d_{1}, \ldots, d_{6}$ & BC & 1.9 & 1.9 \\
\hline$\left\langle r_{1}\right\rangle$ & 0.4 & $d_{4}, d_{5}, d_{6}$ & C & 1.6 & 2.0 \\
\hline$\left\langle r_{1}, r_{3}\right\rangle$ & 0.8 & $d_{4}$ & CD & 0.0 & 1.2 \\
\hline
\end{tabular}

Table 3. The cutoff error for each prefix of $\left\langle r_{1}, r_{3}, r_{4}\right\rangle$

\begin{tabular}{|l|l|l|l|l|l|}
\hline$\langle r 1, \ldots, r i\rangle$ & Error $(r i)$ & unclassified doc. & default classset & default error & cutoff error \\
\hline$\langle>$ & 0 & $d_{1}, \ldots, d_{6}$ & BC & 1.9 & 1.9 \\
\hline$\left\langle r_{1}\right\rangle$ & 0.4 & $d_{4}, d_{5}, d_{6}$ & C & 1.6 & 2.0 \\
\hline$\left\langle r_{1}, r_{2}\right\rangle$ & 0.75 & $d_{5}, d_{6}$ & C & 0.8 & 1.95 \\
\hline$\left\langle r_{1}, r_{2}, r_{3}\right\rangle$ & 0.8 & & & 0 & 1.95 \\
\hline
\end{tabular}

Table 4. The cutoff error for each prefix of $\left\langle r_{1}, r_{2}, r_{3}, r_{4}\right\rangle$

within the $p$ weight percentile. These are the classes on the top of the list whose total weight is equal to $p$ percentage of the total weight of the whole list. For all methods, the error on a testing document is measured by Equation (1). All results are the average of the 5 -fold cross-validation.

\subsection{The data sets}

The IBM Patent data set (http://www.patents.ibm.com/patlist?xcl=0/). This database contains patent documents categorized by branches and sub-branches. We use branch 451 (Abrading) with 39 sub-branches, and branch 051 (Abrasive tool making process, material, or composition) with 14 sub-branches. For each patent document, we use terms only in Title, Inventor, Abstract and Current class. A class has the form of branch/sub-branch. For example, 451/430 denotes the class corresponding to branch 451 and sub-branch 430. Most documents are associated with one class, and the rest are associated with two or more classes.

The ACM data set (http://www.acm.org/dl/toc.html). This data set maintains a 4-level hierarchical classification of computing related papers. We use level-1 and level-2 topics as the class hierarchy and add level- 3 and level-4 topics as terms to documents. Each document is associated with the set of level-1 and level-2 topics of the document. We remove the documents whose classsets appear in less than 15 documents.

Table 5 shows some statistics of the two data sets after the above processing. The partitioning of training documents and testing documents is determined by the 5 -fold cross validation. 


\begin{tabular}{|l|c|c|}
\hline & ACM & IBM Patent \\
\hline Documents & 15981 & 4974 \\
\hline Classsets & 288 & 191 \\
\hline classes & 40 & 21 \\
\hline terms & 9590 & 14991 \\
\hline level of class hierarchy & 3 & 3 \\
\hline average size of documents & 10.80 & 45 \\
\hline average size of classsets & 1.86 & 1.55 \\
\hline training documents & 12784 & 3979 \\
\hline testing documents & 3197 & 995 \\
\hline
\end{tabular}

Table 5. The statistics of the processed data sets

\subsection{The result on IBM Patent data set}

Figure 2 shows the classification error over the 995 testing documents. For example, the error of 220 means that the average error of classifying each of the 995 testing documents is $220 / 995=0.22$, which is the difference of the observed classset and the predicted classset, or the difference of the documents belonging to these classsets. On the left side is the error of Coverage and Confidence. The $\mathrm{x}$-axis denotes the minimum support for mining association rules. Different figures correspond to different accuracy thresholds for selecting rules in Step 3. On the right side is the error of $\mathrm{kNN}$. The $\mathrm{x}$-axis denotes the parameter $k$. Different figures correspond to different feature thresholds. Different curves correspond to different cutoff thresholds of class list. Several observations follow.

The error. Coverage performs significantly better than Confidence, i.e., reducing the error up to $67 \%$. Two factors contribute to this difference. First, Coverage searches for all rules determining a subset of the classset in a training document, but Confidence does not because it treats each classset in the training documents as a new class. As a result, Confidence generates few rules that satisfy the given minimum support, which can be seen from Figure 3, and classification often is done by the default rule. Another reason is that Confidence ignores the similarity of classes, thus, makes no attempt to assign a document to a more similar class in the case of misclassification. The experiment also shows that the error of Coverage is sensitive to the minimum support, but not to the accuracy threshold. Using a small minimum support, Coverage is about $10 \%$ to $30 \%$ better than the best kNN result.

The size of classifier. On the left side of Figure 3 is the size of the classifiers constructed by Coverage and Confidence. For both methods, the minimum support and the accuracy threshold affects the size. The experiment suggests that minimum support of $1 \%$ and accuracy threshold of $2 \%$ give a classifier that is both accurate and small.

The execution time. As shown on the right side of Figure 3, Coverage takes longer time than Confidence due to computing the similarity between classsets. The experiments shows that the minimum support of $1 \%$ is good for both accuracy and 
speed.

\subsection{The result on the ACM data set}

The error on the ACM data set is shown in Figure 4. The error is measured over 3197 testing documents. The comparison is consistent with that using the IBM Patent data. The best accuracy of Coverage is typically $30 \%$ to $50 \%$ higher than of that of kNN.

\section{Conclusion}

In real life, the class space of documents is a specific-to-general hierarchy and a document may belong to more than one class in the hierarchy. In this paper, an automatic classification of documents with this feature was proposed. In this setting, classes are no longer independent of each other in that they classify some documents in common, and those that classify more documents in common should be considered as more similar to each other than those that classify few documents in common. A notion of similarity of classsets based on the similarity of the documents classified by classsets was proposed to capture this reality. An algorithm for constructing a classifier based on this notion of class similarity was presented. Experiments on real life datasets show that the proposed method achieves much higher accuracy than traditional classifiers. 


\section{Bibliography}

[1] R. Agrawal, T. Imielinski, and A. Swami, Mining Association Rules between Sets of Items in Large Databases, SIGMOD 1993

[2] R. Agrawal and R. Srikant, Fast Algorithms for Mining Association Rules, VLDB 1994

[3] S. Chakrabarti, D. Dom, R. Agrawal, and P. Raghavan, Using Taxonomy, Discriminants, and Signatures for Navigating in Text Databases, VLDB 1997.

[4] D. Koller and M. Sahami, Hierarchically Classifying Documents Using Very Few Words, International Conference on Machine Learning, 1997

[5] J.R. Quinlan, C4.5: Programs for Machine Learning, Morgan Kaufmann, 1993

[6] H. Schutze, D.A. Hull, and J.O. Pederson, A Comparison of Classifiers and Document Representations for the Routing Problem, SIGIR 1995, 229-237

[7] K. Wang, S. Zhou, S.C. Liew, Building Hierarchical Classifiers Using Class Proximity, VLDB 1999

[8] Y. Yang and C.G. Chute, A Linear Least Squares Fit Mapping Method for Information Retrieval from Natural Language Texts, COLING-92, 1992

[9] Y. Yang and J.O. Pederson, A Comparative Study on Feature Selection in Text Categorization, International Conference on Machine Learning 1997. 

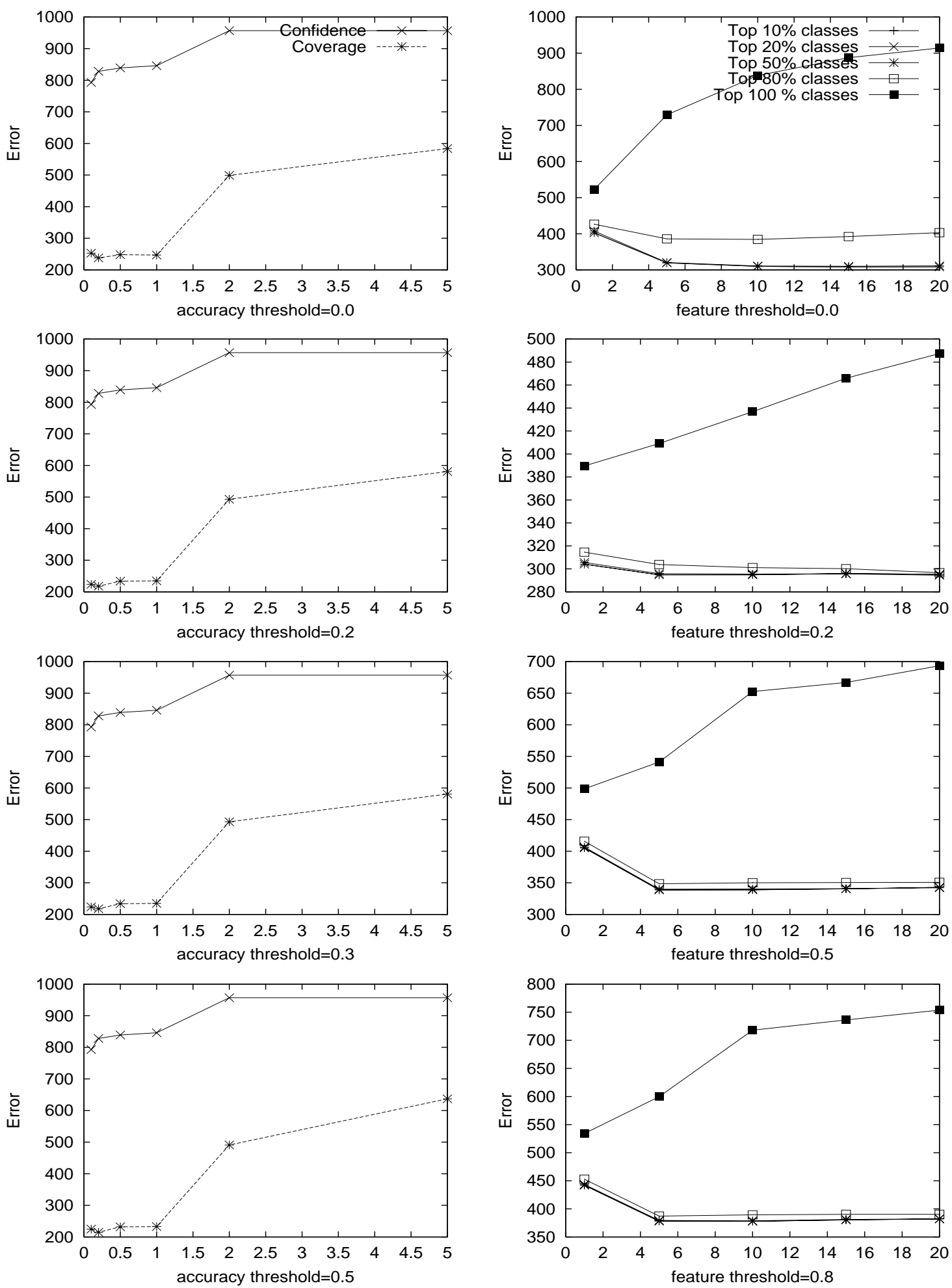

Figure 2. Patent data set: Coverage and Confidence (left) and kNN (right) 

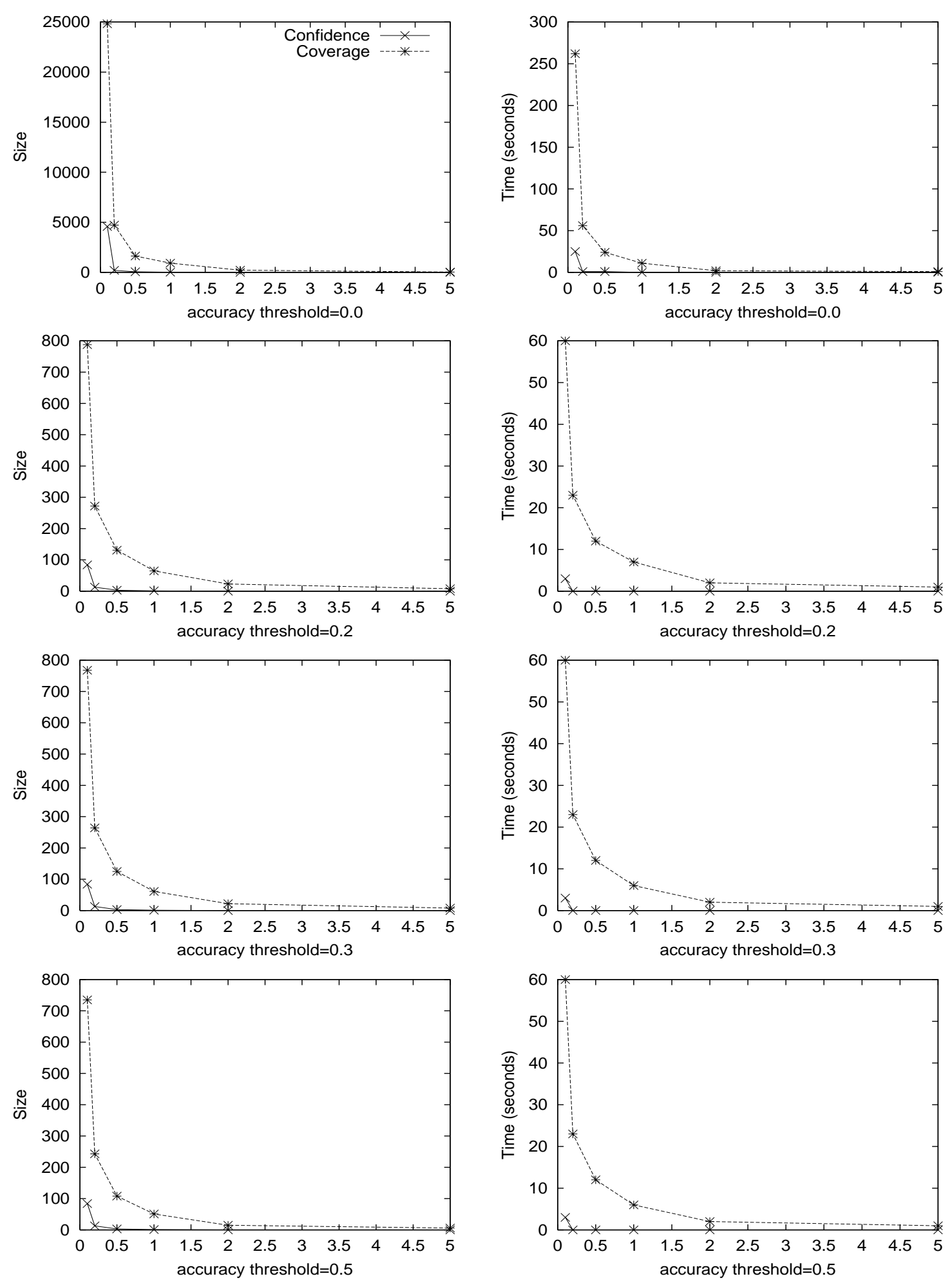

Figure 3. Patent data set: classifier size (left) and execution time 

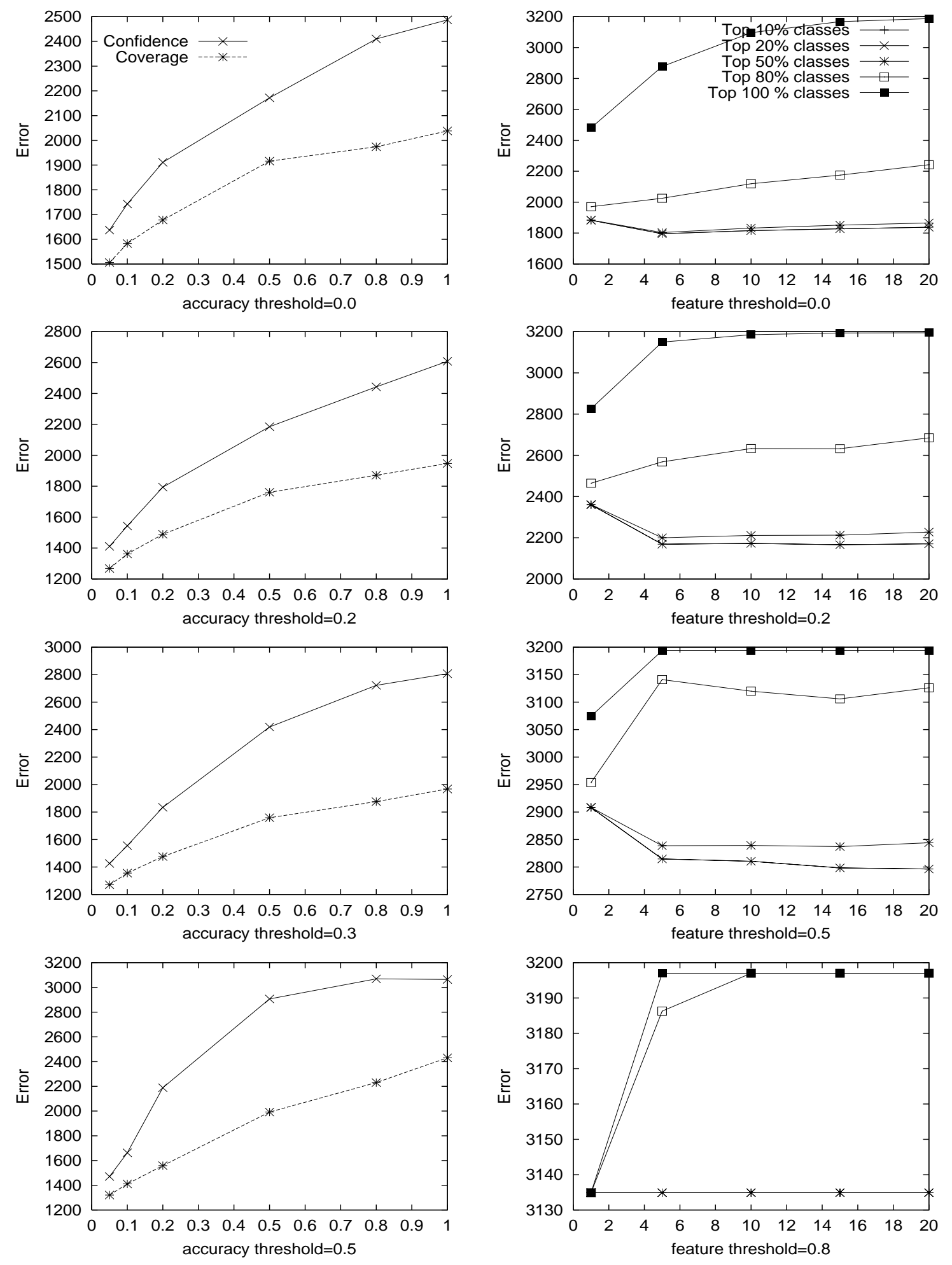

Figure 4. ACM data set: Coverage and Confidence (left) and kNN (right) 